\title{
WEIGHTED NORM ESTIMATES FOR THE FOURIER TRANSFORM WITH A PAIR OF WEIGHTS
}

\author{
JAN-OLOV STRÖMBERG AND RICHARD L. WHEEDEN
}

\begin{abstract}
We prove weighted norm inequalities of the form

$$
\|\hat{f}\|_{L_{u}^{q}} \leq C\|f\|_{H_{v}^{p}}, \quad 0<p \leq q<\infty,
$$

for the Fourier transform on $\mathbf{R}^{n}$. For some weight functions $v$, the Hardy space $H_{v}^{p}$ on the right can be replaced by $L_{v}^{p}$. The proof depends on making an atomic decomposition of $f$ and using cancellation properties of the atoms.
\end{abstract}

\section{INTRODUCTION.}

As indicated by Pitt's theorem (see Stein [St]), the following weighted inequality holds for the Fourier transform of a function $f$ on $R$ :

$$
\int_{R}|\hat{f}(y)|^{p}|y|^{p-2-\gamma} d y \leq C \int_{R}|f(x)|^{p}|x|^{\gamma} d x
$$

when $1<p<\infty$ and $\max (0, p-2) \leq \gamma<p-1$ (and also a similar estimate with an exponent $q>p$ on the left-hand side). Sadosky and Wheeden show in [Sad-W] that the inequality above holds for all $\gamma \geq \max (0, p-2)$ with $\gamma \neq k p-1, k=1,2, \ldots$, if we assume in addition that the function $f$ has vanishing moments up to a certain order depending on $\gamma$ and $p$; in fact they show that if $1<p \leq q<\infty, k$ is a positive integer and the function $f$ has vanishing moments of order less than or equal to $k-1$ then

$$
\left(\int_{R}\left(\frac{|\hat{f}(y)|}{|y|^{k-1}}\right)^{q}\left(\frac{1}{|y|} w\left(\frac{1}{y}\right)\right)^{q / p} \frac{d y}{|y|}\right)^{1 / q} \leq C\left(\int_{R}|f(x)|^{p}|x|^{k p} w(x) d x\right)^{1 / p}
$$

for any weight $w$ satisfying $w^{q / p} \in A_{1+\left(q / p^{\prime}\right)}, 1 / p+1 / p^{\prime}=1$. (See $\S 2$ for the definition of $A_{p}$.) In fact a slightly weaker condition on $w$ is sufficient. Other results of this kind are given in Benedetto, Heinig and Johnson [B-H-J] and in Benedetto and Heinig [B-H].

We see that the origin plays a special role in the description of the weights in the inequalities above. This may be natural on the Fourier transform side since

Received by the editors March 3, 1989.

1980 Mathematics Subject Classification (1985 Revision). Primary 42B10; Secondary 42B30.

Supported in part by NSF grant DMS 87-03546. 
we assume that $\hat{f}(\xi)$ vanishes at the origin to a certain order. However, on the function side the origin should not play any more role than any other point.

In this paper we consider conditions for a pair $(u, v)$ of weights such that the $L_{u}^{q}$ norm of the Fourier transform is dominated by the weighted Hardy space $H_{v}^{p}$ norm. The application we have in mind is the case when $v(x)=$ $|Q(x)|^{p} w(x)$, where $Q$ is a polynomial and $w \in A_{p}$. If a function $f$ has vanishing moments up to a certain order depending on $Q$ then the $H_{v}^{p}$ norm of $f$ is dominated by its $L_{v}^{p}$ norm. This will give us an estimate

$$
\|\hat{f}\|_{L_{u}^{q}} \leq C\|f\|_{L_{v}^{p}}
$$

which is a generalization of the inequality obtained by Sadosky and Wheeden.

Note that the estimates of the Fourier transform which have been obtained by Jurkat and Sampson [J-Sam], Muckenhoupt [M], and Heinig [H1] are quite different, since the condition there is stated in terms of rearrangements of the weights, and moment conditions do not enter their estimates. See also [B-H-J and $\mathrm{H} 2$ ] for some further results.

The weighted Hardy space norm inequality in this paper is done in $R^{n}$ and the proof uses Hausdorff-Young's inequality and depends heavily on the atomic decomposition of Hardy spaces. In $\S 2$ we give some preliminaries which are used later. In $\S 3$ we state the main result with the condition on the weights. In $\S \S 4$ and 5 we make the technical estimate of the Fourier transform for a finite sum of atoms, and in $\S 6$ we use some rather simple limit arguments to obtain our main result from the estimates which are proved in the previous section.

\section{Preliminaries}

We now introduce some notation. The halfspace $R_{+}^{n+1}$ is the set $R_{+}^{n+1}=$ $\left\{(x, t): x \in R^{n}\right.$ and $\left.t>0\right\}$ and the ball $B(x, t)$ in $R^{n}$ is the set $B(x, t)=$ $\left\{y \in R^{n}:|y-x|<t\right\}$. The Lebesgue measure of a set $E$ in $R^{n}$ is denoted by $|E|$. A weight function (or a weight) $w$ on $R^{n}$ is a nonnegative function on $R^{n}$. By $w(E)$ we denote the corresponding measure of the set $E$ in $R^{n}$, i.e., $w(E)=\int_{E} w(x) d x$. Let $1 \leq d<\infty$. The weight function $w$ satisfies the doubling condition $D_{d}$ (i.e., $w \in D_{d}$ ) if

$$
w(B(x, r t)) \leq C r^{n d} w(B(x, t)) \text { for all } t>0, r \geq 1 \text {, and } x \in R^{n},
$$

with the constant $C$ independent of $t, r$, and $x$. We say that $w \in D_{\infty}$ if $w \in D_{d}$ for some $d \geq 1$. Let $\psi$ be a function in the Schwartz class $\mathscr{S}$, and let $\psi_{t}(x)=\psi(x / t) t^{-n}$. The nontangential maximal function $N_{\psi} f$ of a tempered distribution $f$ is defined by $N_{\psi} f(x)=\sup _{(y, t):|y-x|<t}\left|f * \psi_{t}(y)\right|$. For $\lambda>0$, we define the tangential maximal function $N_{\psi}^{\lambda} f$ by

$$
N_{\psi}^{\lambda} f(x)=\sup _{(y, t) \in R_{+}^{n+1}}\left|f * \psi_{t}(y)\right|(1+|x-y| / t)^{-\lambda},
$$


and the grand maximal function $f^{*}=f^{* M}$ by $f^{* M}(x)=\sup _{\psi} N_{\psi} f(x)$, where the supremum is taken over all Schwartz functions with Schwartz norm

$$
\|\psi\|_{M}=\sup _{x \in R^{n},|\alpha| \leq M}\left|D^{\alpha} \psi(x)\right|(1+|x|)^{M} \leq 1 .
$$

The weighted Hardy space $H_{w}^{p}$ is defined as the space of tempered distributions $f$ with norm $\|f\|_{H_{w}^{p}}=\left\|N_{\psi} f\right\|_{L_{w}^{p}}<\infty$, where $\psi$ is a function in $\mathscr{S}$ with $\int \psi \neq 0$ and $\|\cdot\|_{L_{w}^{p}}$ is defined by $\|g\|_{L_{w}^{p}}=\left(\int_{R^{n}}|g(x)|^{p} w(x) d x\right)^{1 / p}$, $0<p<\infty$. Observe by [Str-T] that if $w \in D_{\infty}$ this definition is independent of $\psi$ with norm within a constant factor. It would also be equivalent to use the tangential maximal function $N_{\psi}^{\lambda} f$ or the grand maximal function $f^{* M}$ instead of $N_{\psi} f$ when defining the weighted Hardy space norm provided that $\lambda$ or $M$, respectively, is large enough depending on the doubling exponent $d$ and on $p$. In fact, $N_{\psi}^{\lambda} f(x) \leq C N_{\psi_{1}}^{\lambda} f(x)$ for any two functions $\psi$ and $\psi_{1}$ in $\mathscr{S}$ with nonzero integrals, with the constant $C$ depending only on $\psi, \psi_{1}$, and $\lambda$, and similarly $N_{\psi}^{\lambda} f(x) \leq C f^{* M}(x)$ when $\lambda \geq M$ and $f^{* M}(x) \leq C N_{\psi}^{\lambda} f(x)$ when $M>M(\lambda)$.

If $p>1$ and the weight $w$ satisfies Muckenhoupt's $A_{p}$ condition:

$$
\frac{1}{|B|} \int_{B} w d x\left(\frac{1}{|B|} \int_{B} w^{-\frac{1}{p-1}} d x\right)^{p-1} \leq C \text { for all balls } B
$$

then the $H_{w}^{p}$ norm and the $L_{w}^{p}$ norm are equivalent, and the spaces $H_{w}^{p}$ and $L_{w}^{p}$ can be identified in the natural way:

$$
\langle f, \psi\rangle=\int f(x) \psi(x) d x \text { for } \psi \text { in } \mathscr{S} .
$$

In this paper, however we mainly consider $H_{v}^{p}$ spaces where the weight $v$ does not satisfy the $A_{p}$ condition. One type of weight we have in mind is the following.

Let $v$ be a weight on $R$ which can be written $v(x)=|Q(x)|^{p} w(x)$ with $w \in$ $A_{p}, p>1$, and $Q$ a polynomial of nonzero degree. Then $v$ does not satisfy the $A_{p}$ condition and the spaces $H_{v}^{p}$ and $L_{v}^{p}$ are not equal under the identification described above. However, $H_{v}^{p}$ can still be identified with a subspace of $L_{v}^{p}$ defined by some moment conditions (in fact, the subspace is the whole space $L_{v}^{p}$ if $Q$ has only real zeros). We refer to Strömberg-Wheeden [Str-W]. (A similar identification between weighted Hardy and Lebesgue spaces holds in higher dimensions for $Q$ generalized in a suitable way. (See Adams [A].)) This identification of a function with a tempered distribution coincides with the natural one in the case when the function is locally integrable and has zero moments of order less than the degree of $Q$, e.g., for functions in the class $\mathscr{S}_{0,0}$, which is defined as the space of functions in $\mathscr{S}$ whose Fourier transforms are compactly supported away from the origin. 
For a function $f$ in $\mathscr{S}$ we define the Fourier transform by

$$
\hat{f}(y)=\int_{R^{n}} f(x) e^{-2 \pi i x \cdot y} d x
$$

and for a tempered distribution $f$ we define its Fourier transform $\hat{f}$ to be the tempered distribution defined by $\langle\hat{f}, \varphi\rangle=\langle f, \hat{\varphi}\rangle$ for all $\varphi \in \mathscr{S}$.

\section{MAIN RESULT}

First we introduce a weight condition for a pair $(u, v)$ of weights. Let $1 \leq$ $p_{1} \leq 2,1 / p_{1}+1 / q_{1}=1,0<p \leq q<\infty$ and $q \leq q_{1}$. Let $\varphi$ be a nonnegative compactly supported function on $R^{n}$ which is not identically zero, and let $\varphi_{t}(x)=t^{-n} \varphi(x / t)$. A pair of weights $u$ and $v$, with $v$ satisfying the doubling condition, is said to satisfy the $P W\left(q, p ; q_{1}, p_{1}\right)$ condition if there is a constant $C$ such that for all $t>0$

$$
\left(\int_{|y| \geq t} u^{\frac{q_{1}}{q_{1}-q}} d y\right)^{\frac{q_{1}-q}{q_{1} q}}\left(\int_{R^{n}}\left(\varphi_{1 / t} * v\right)^{-\frac{p_{1}}{p-p_{1}}} d x\right)^{\frac{p-p_{1}}{p_{1} p}} \leq C
$$

in the case $p_{1}<p$, or

$$
\left(\int_{|y| \geq t} u^{\frac{q_{1}}{q_{1}-q}} d y\right)^{\frac{q_{1}-q}{q_{1} q}}\left(t^{-\frac{n\left(p_{1}-p\right)}{p_{1}}} \inf _{x \in R^{n}}\left(\varphi_{1 / t} * v(x)\right)\right)^{-\frac{1}{p}} \leq C
$$

in the case $p_{1} \geq p$. (When $q_{1}=q$ the left expression means $\left(\sup _{|y| \geq t} u(y)\right)^{1 / q}$ and when $q_{1}=\infty$ it means $\left(\int_{|y|>t} u d y\right)^{1 / q}$.)

The notation " $P W$ " used above is an abbreviation for "pair of weights". We note that the condition allows the consideration of weights $v$ which have zeros of large order; e.g., if $v(x)=|x|^{\gamma}, \gamma>-n$, it is easy to see that

$$
\varphi_{1 / t} *|x|^{\gamma} \approx(|x|+1 / t)^{\gamma}
$$

and for $r>0$,

$$
\left(\int_{R^{n}}\left(\varphi_{1 / t} *|x|^{\gamma}\right)^{-r} d x\right)^{1 / r} \approx \begin{cases}t^{\gamma-n / r} & \text { if } \gamma>n / r \\ \infty & \text { otherwise. }\end{cases}
$$

Furthermore, if $v(x)=|x|^{k p} w(x)$ for $w \in A_{p}, k=1,2, \ldots$, and $1<p<$ $\infty$, then

$$
\left(\int_{R^{n}}\left(\varphi_{1 / t} * v(x)\right)^{-\frac{1}{p-1}} d x\right)^{p-1} \approx\left(\int_{|x|>1 / t} w(x)^{-\frac{1}{p-1}} \frac{d x}{|x|^{k p^{\prime}}}\right)^{p-1}
$$

$1 / p+1 / p^{\prime}=1$. If $k \geq n$, this expression is finite and equivalent to

$$
t^{(k-n) p}\left(\int_{|x|<1 / t} w(x) d x\right)^{-1} .
$$


Weights $v$ of this kind were considered in [Sad-W] and are naturally related to the $P W(p, q ; \infty, 1)$ condition, i.e., to the condition

$$
\left(\int_{|x|>t} u d x\right)^{\frac{1}{q}}\left(\int_{R^{n}}\left(\varphi_{1 / t} * v(x)\right)^{-\frac{1}{p-1}} d x\right)^{\frac{p-1}{p}} \leq C,
$$

$1<p<\infty$. In fact, it is not difficult to see that the weights in [Sad-W] satisfy this condition.

The condition $P W\left(q, p ; q_{1}, p_{1}\right)$ is also of interest for values of $p_{1}$ other than 1 : e.g., the classical result of Hardy that $\|\hat{f}(x) /|x|\|_{L^{1}} \leq C\|f\|_{H^{1}}$ when $n=1$ corresponds to the case $p_{1}=q_{1}=2, p=q=1, u=1 /|x|$, and $v=1$; note that $\left(1^{\prime}\right)$ is the appropriate condition to check in this case since $p_{1}>p$.

Remark. The integral expression

$$
\left(\int_{R^{n}}\left(\varphi_{1 / t} * v\right)^{-\frac{p_{1}}{p-p_{1}}} d x\right)^{\frac{p-p_{1}}{p_{1} p}} \quad\left(\text { when } p_{1}<p\right)
$$

may also be interpreted as a sum

$$
\left(\sum_{Q_{k}}\left|Q_{k}\right| \frac{v\left(Q_{k}\right)^{-\frac{p_{1}}{p-p_{1}}}}{\left|Q_{k}\right|}\right)^{\frac{p-p_{1}}{p_{1} p}}
$$

where the sum is taken over all cubes $Q_{k}$ with side $1 / t$ in a dyadic decomposition of $R^{n}$. Similarly, $\inf _{x \in R^{n}}\left(\varphi_{1 / t} * v(x)\right)$ in the case $p_{1} \geq p$ can be interpreted as $\inf _{y \in R^{n}} v\left(B_{1 / t}(y)\right) /\left|B_{1 / t}(y)\right|$. In the case $p_{1}<p$ the $P W\left(q, p ; q_{1}, p_{1}\right)$ condition can be written

$$
\left(\int_{|y| \geq t} u^{\frac{q_{1}}{q_{1}-q}} d y\right)^{\frac{q_{1}-q}{q_{1} q}} \leq C\left(\sum_{s\left(Q_{k}\right)=1 / t}\left|Q_{k}\right|\left(\frac{v\left(Q_{k}\right)}{\left|Q_{k}\right|}\right)^{-\frac{p_{1}}{p-p_{1}}}\right)^{-\frac{p-p_{1}}{p_{1} p}} \quad \text { for all } t>0,
$$

where the sum is taken over all cubes $Q_{k}$ in the dyadic decomposition of $R^{n}$ into cubes $Q_{k}$ with side $s\left(Q_{k}\right)=1 / t$. In the case $p_{1} \geq p$ the $P W\left(q, p ; q_{1}, p_{1}\right)$ condition can be written

$$
\left(\int_{|y| \geq t} u^{\frac{q_{1}}{q_{1}-q}} d y\right)^{\frac{q_{1}-q}{q_{1} q}} \leq C\left(t^{-\frac{n\left(p_{p}-p\right)}{p_{1}}} \inf _{y \in R^{n}}\left(v\left(B_{1 / t}(y)\right) /\left|B_{1 / t}(y)\right|\right)\right)^{\frac{1}{p}} \quad \text { for all } t>0 .
$$

Our main result is the following weighted estimate for the Fourier transform.

Theorem 1. Let $0<p \leq q<\infty$ and let $(u, v)$ be a pair of weights with $v$ satisfying the doubling condition and the pair $(u, v)$ satisfying the $P W\left(q, p ; p_{1}, q_{1}\right)$ condition for some $1 \leq p_{1} \leq q_{1} \leq \infty, 1 / p_{1}+1 / q_{1}=1$, with $q \leq q_{1}$. Then

$$
\|\hat{f}\|_{L_{u}^{q}} \leq C\|f\|_{H_{v}^{p}}
$$

for all tempered distributions $f$ in $H_{v}^{p}$. 
Remark 1. The doubling property of the weight $v$ is used to get an intrinsic definition of the $H_{v}^{p}$ space norm and then mainly used to obtain the left-hand side inequality in Lemma 1 below, namely,

$$
\frac{1}{C}\left(\frac{s}{t}\right)^{n(d-1) r} \int_{R^{n}}\left(\varphi_{s} * v(x)\right)^{-r} d x \leq \int_{R^{n}}\left(\varphi_{t} * v(x)\right)^{-r} d x \text { for } s \leq t .
$$

If we only assume that this holds for some $d$ instead of the somewhat stronger condition that $v$ is doubling, then we obtain, for any $\lambda>0$, the inequality

$$
\left(\int_{R^{n}}|\hat{f}(y)|^{q} u(y) d y\right)^{1 / q} \leq C\left(\int_{R^{n}}\left|N_{\psi}^{\lambda} f(x)\right|^{p} v(x) d x\right)^{1 / p} \quad \text { for } f \in \mathscr{S}_{0,0} .
$$

Remark 2. In the condition on the pair of weights $(u, v)$ given in the theorem it is a problem to choose the exponents $p_{1}$ and $q_{1}$ in an optimal way. By splitting $u$ into parts $u=u_{1}+u_{2}$ and using different pairs of exponents $\left(q_{1}, p_{1}\right)$ for $u_{1}$ and $u_{2}$, we get a weaker condition on the weights $u$ and $v$. We will not go further into this problem.

Remark 3. If we want to consider the limiting case $q=\infty$ we should first replace the weight $u$ by $u^{q}$, both in the $P W$ condition and in the weighted norms before we let $q$ go to infinity. Then we get

$$
\sup _{y \in R^{n}}|\hat{f}(y)| u(y) \leq C\|f\|_{H_{v}^{p}}
$$

when $0<p<\infty$ with the $P W$ condition

$$
\sup _{|y|>t} u(y) \leq C \begin{cases}\left(\int_{R^{n}}\left(\varphi_{1 / t} * v(x)\right)^{-\frac{1}{p-1}} d x\right)^{-\frac{p-1}{p}} & \text { for } p>1, \\ \left(t^{-n(1-p)} \inf _{x \in R^{n}}\left(\varphi_{1 / t} * v(x)\right)\right)^{1 / p} & \text { for } 0<p \leq 1 .\end{cases}
$$

If we also replace the weight $v$ by $v^{p}$ and consider the limiting case when $p=\infty$ we get

$$
\sup _{y \in R^{n}}|\hat{f}(y)| u(y) \leq C \sup _{x \in R^{n}} N_{\psi}^{\lambda} f(x) v(x)
$$

provided

$$
\sup _{|y|>t} u(y) \leq C\left(\int_{R^{n}}\left(\sup _{|z-x| \leq c / t} v(z)\right)^{-1} d x\right)^{-1} .
$$

We leave the details to the reader.

Let $p>1$ and assume that the weight $v$ on $R$ can be written $v(x)=$ $|Q(x)|^{p} w(x)$ where $Q$ is a polynomial and $w \in A_{p}$. Then $\|f\|_{H_{v}^{p}} \leq C\|f\|_{L_{v}^{p}}$ for all $f \in \mathscr{S}_{0,0}$ (see [Str-W]) and we get the following corollary.

Corollary. Let $1<p \leq q<\infty$ and let $v=|Q|^{p} w$ where $Q$ is a polynomial on $R$ and $w$ is a weight on $R$ satisfying the $A_{p}$ condition. Assume also that 
the pair of weights $(u, v)$ satisfies the $P W\left(q, p, ; q_{1}, p_{1}\right)$ condition for some $p_{1}$ and $q_{1}, 1 / p_{1}+1 / q_{1}=1$ with $1 \leq p_{1} \leq 2$ and $q_{1} \geq q$. Then

$$
\|\hat{f}\|_{L_{u}^{q}} \leq C\|f\|_{L_{v}^{p}} \text { for all } f \in \mathscr{S}_{0,0} .
$$

\section{TeCHNICAL PROOF}

We are going to use the atomic decomposition of $f \in H_{v}^{p}$. The space $\mathscr{S}_{0,0}$ is dense in $H_{v}^{p}$ and a function in $\mathscr{S}_{0,0}$ can be approximated by finite sums of atoms. In this section we state and prove an estimate for the Fourier transform of a finite sum of atoms. In a later section this estimate will be extended to $\mathscr{S}_{0,0}$ and $H_{v}^{p}$ by rather simple limiting arguments.

Let $f$ be a finite sum $\sum \lambda_{k} a_{k}$ where $\lambda_{k}$ are nonnegative numbers and $\left\{a_{k}\right\}$ are atoms satisfying

$\left|a_{k}(x)\right| \leq 1$,

$\int_{R^{n}} a_{k}(x) x^{\alpha} d x=0$, for all $|\alpha| \leq m$,

$a_{k}$ is supported in a cube $Q_{k}$.

Set $f^{* *}=\sum \lambda_{k} \chi_{Q_{k}}$ and, more generally, for $0<r<\infty$, set $f^{* r *}=\left(\sum \lambda_{k}^{r} \chi_{Q_{k}}\right)^{\frac{1}{r}}$. We are going to show

Proposition 1. Let $0<p \leq q<\infty$. Let $u$ and $v$ be weights on $R^{n}$ with $v \in D_{d}$ and suppose that $u$ and $v$ satisfy the $P W\left(q, p ; q_{1}, p_{1}\right)$ condition for some $p_{1}, q_{1}, 1 \leq p_{1} \leq 2, q_{1} \geq q$, and $1 / p_{1}+1 / q_{1}=1$.

If $q \geq 1$ then we have

$$
\left(\int_{R^{n}}|\hat{f}(y)|^{q} u(y) d y\right)^{1 / q} \leq C\left(\int_{R^{n}}\left|f^{* *}(x)\right|^{p} v(x) d x\right)^{1 / p}
$$

and more generally, if $0<r \leq 1$ and $q \geq r$ then we have

$$
\left(\int_{R^{n}}|\hat{f}(y)|^{q} u(y) d y\right)^{1 / q} \leq C\left(\int_{R^{n}}\left|f^{* r *}(x)\right|^{p} v(x) d x\right)^{1 / p}
$$

for finite sums $f$ of atoms with vanishing moments of order less than or equal to $m$, provided $m>-1+n\left[d-\min \left(1, p / p_{1}\right)\right] / p$.

The proof of Theorem 1 is based on Hausdorff-Young's inequality:

$$
\|\hat{g}\|_{L^{q_{1}}} \leq C_{p_{1}}\|g\|_{L^{p_{1}}}
$$

for $1 \leq p_{1} \leq 2$ and $1 / p_{1}+1 / q_{1}=1$.

First we are going to break the sum $f$ of atoms into pieces $f_{j}$ according to the sizes of the corresponding cubes. We need

Lemma 1. Let $v(x)$ be a weight in $D_{d}$ and let $\varphi_{t}(x)=\varphi(x / t) t^{-n}$ where $\varphi$ is a nonnegative compactly supported $C^{\infty}$ function with $\int \varphi=1$. Then for $r>0$ there is a constant $C$ depending only on $\varphi$ and $r$ such that

$$
\begin{aligned}
\frac{1}{C}\left(\frac{s}{t}\right)^{n(d-1) r} \int_{R^{n}}\left(\varphi_{s} * v(x)\right)^{-r} d x & \leq \int_{R^{n}}\left(\varphi_{t} * v(x)\right)^{-r} d x \\
& \leq C \int_{R^{n}}\left(\varphi_{s} * v(x)\right)^{-r} d x
\end{aligned}
$$


for all $t \geq s>0$; and in the limit case $r=\infty$ we have

$$
C\left(\frac{t}{s}\right)^{n(d-1)} \inf _{x \in R^{n}} \varphi_{s} * v(x) \geq \inf _{x \in R^{n}} \varphi_{t} * v(x) \geq \frac{1}{C} \inf _{x \in R^{n}} \varphi_{s} * v(x)
$$

for all $t \geq s>0$.

Proof of Lemma 1. Using the doubling property of the weight $v$ we get $\varphi_{t} *$ $v(x) \sim v(B(x, t)) /|B(x, t)|$ and from this and the $D_{d}$ condition we see that

$$
\frac{1}{C} \varphi_{s} * \varphi_{t} * v(x) \leq \varphi_{t} * v(x) \leq C\left(\frac{t}{s}\right)^{n(d-1)} \varphi_{s} * v(x) \text { for } s \leq t .
$$

From this we get the limit case $r=\infty$ and the left inequality of the lemma in the case $r<\infty$. For the right inequality in the case $r<\infty$ we use Jensen's inequality to obtain $\varphi_{t} *\left(\left(\varphi_{s} * v\right)^{-r}\right)(x) \geq\left(\varphi_{t} * \varphi_{s} * v(x)\right)^{-r}$ and by integration

$$
\begin{aligned}
& \int_{R^{n}}\left(\varphi_{t} * v(x)\right)^{-r} d x \leq C \int_{R^{n}}\left(\varphi_{t} * \varphi_{s} * v(x)\right)^{-r} d x \\
& \quad \leq C \int_{R^{n}} \varphi_{t} *\left(\left(\varphi_{S} * v\right)^{-r}\right)(x) d x=C \int_{R^{n}}\left(\varphi_{s} * v(x)\right)^{-r} d x
\end{aligned}
$$

By Lemma 1, we see that if $p>p_{1}$ and $\int_{R^{n}}\left(\phi_{1 / s} * v(x)\right)^{-p_{1} /\left(p-p_{1}\right)} d x$ is infinite for one value of $s$ then it is infinite for all values of $s$. In this case, if the pair $(u, v)$ satisfies the $P W\left(q, p ; q_{1}, p_{1}\right)$ condition for any weight $u$ then $u$ must be identically zero on $R^{n}$, and consequently, Theorem 1 is trivially true. If $p>p_{1}$ and the integral is finite, we can, by Lemma 1 , partition $(0, \infty)$ into intervals with endpoints $s_{j}$ such that $s_{j}<s_{j+1}$ and

$$
\left(\int_{R^{n}}\left(\phi_{1 / s} * v(x)\right)^{-\frac{p_{1}}{p-p_{1}}} d x\right)^{\frac{p-p_{1}}{p_{1}}} \sim \beta^{j} \quad \text { for } s_{j}<s<s_{j+1},
$$

where $\beta>1$ is a constant depending on the constant $C$ in Lemma 1. The sequence $\left\{s_{j}\right\}$ might be finite, infinite to the left or to the right, or infinite at both ends. The equivalence above holds for the endpoints $s=s_{j}$ and $s=$ $s_{j+1}$ provided $0<s<\infty$. Furthermore it follows from Lemma 1 that $s_{j} \geq$ $C \beta^{(j-k) / n(d-1)} s_{k}$ for $j>k$.

To be more precise, if we set

$$
F(s)=\left(\int_{R^{n}}\left(\varphi_{1 / s} * v(x)\right)^{-\frac{p_{1}}{p_{-1}}} d x\right)^{\frac{p-p_{1}}{p_{1}}}, \quad 0<s<\infty,
$$

then by Lemma 1

$$
C^{-1} F(s) \leq F(t) \leq C(t / s)^{n(d-1)} F(s), \quad 0<s \leq t<\infty .
$$

Let $\beta=2 C^{2}$ and define $\left\{\sigma_{j}\right\}_{-\infty}^{\infty}$ by

$$
\sigma_{j}=\sup \left\{s: s>0, F(s)<\beta^{j}\right\},
$$


with the convention that $\sigma_{j}=0$ if $\left\{s: s>0, F(s)<\beta^{j}\right\}$ is empty. Then it is not difficult to see that

(a) $\sigma_{j} \leq \sigma_{j+1}$,

(b) $\sigma_{j}<\sigma_{j+1}$ unless $\sigma_{j}=\infty$ or $\sigma_{j+1}=0$,

(c) $C^{-2} \beta^{j} \leq F(s) \leq C^{2} \beta^{j+1}$ if $\sigma_{j}<s<\sigma_{j+1}$,

(d) $C^{-1} \beta^{j} \leq F\left(\sigma_{j}\right) \leq C \beta^{j}$ if $\sigma_{j} \neq 0, \infty$.

Thus we take $\left\{s_{j}\right\}$ to be the distinct $\sigma_{j}, 0 \leq s_{j} \leq \infty$. Also note that

$$
\lim _{s \rightarrow \infty} F(s)=\lim _{s \rightarrow \infty}\left(\int_{R^{n}}\left(\phi_{1 / s} * v\right)^{-\frac{p_{1}}{p-p_{1}}} d x\right)^{\frac{p-p_{1}}{p_{1}}}=\left(\int_{R^{n}} v^{-\frac{p_{1}}{p-p_{1}}} d x\right)^{\frac{p-p_{1}}{p_{1}}}
$$

when $p>p_{1}$, since by Fatou's Lemma and Jensen's inequality

$$
\begin{aligned}
\int_{R^{n}} v^{-r} d x & \leq \liminf _{s \rightarrow \infty} \int_{R^{n}}\left(\phi_{1 / s} * v\right)^{-r} d x \\
& \leq \limsup _{s \rightarrow \infty} \int_{R^{n}}\left(\phi_{1 / s} * v\right)^{-r} d x \leq \int_{R^{n}} v^{-r} d x
\end{aligned}
$$

for $r>0$. Thus if $s_{j+1}=\infty$ then

$$
\left(\int_{R^{n}} v^{-\frac{p_{1}}{p-p_{1}}} d x\right)^{\frac{p-p_{1}}{p_{1}}} \sim \beta^{j+1} .
$$

Similarly, when $p \leq p_{1}$, if $\inf _{x \in R^{n}}\left(\varphi_{1 / s} * v(x)\right)^{-1}=\infty$ for one value of $s$, then the same is true for every $s$, and Theorem 1 is trivial. Thus, as above, we may assume when $p \leq p_{1}$ that $(0, \infty)$ is partioned into intervals with endpoints $s_{j}$ such that $s_{j}<s_{j+1}$ and

$$
F(s)=\left(s^{-\frac{n\left(p_{1}-p\right)}{p_{1}}} \inf _{x \in R^{n}}\left(\varphi_{1 / s} * v(x)\right)\right)^{-1} \sim \beta^{j} \quad \text { for } s_{j}<s<s_{j+1} .
$$

This equivalence also holds for the endpoints $s=s_{j}$ and $s=s_{j+1}$ provided $0<s<\infty$. In particular, by Lemma 1, we get

$$
\frac{1}{C} \beta^{(j-k) /\left(n\left(d-\frac{p}{p_{1}}\right)\right)} \leq \frac{t}{s} \leq C \beta^{(j-k) /\left(n\left(1-\frac{p}{p_{1}}\right)\right)}
$$

if $j>k, s_{k} \leq s \leq s_{k+1}$, and $s_{j} \leq t \leq s_{j+1}$ provided $0<s \leq t<\infty$. We may replace $t / s$ in the inequality above by $s_{j} / s_{k}$ whenever $0<s_{k}<s_{j}<\infty$. However, when $p<p_{1}$ we get $0<s_{j}<\infty$ for all $j$ as a consequence of the inequality above. When $p=p_{1}$ we may have $s_{j+1}=\infty$. If this is the case we conclude in a similar way as when $p>p_{1}$ that

$$
\lim _{s \rightarrow \infty} F(s)=\left(\inf _{x \in R^{n}} v(x)\right)^{-1} \sim \beta^{j+1} .
$$

We define the index sets $I_{j}$ by

$$
I_{j}=\left\{k: 1 / s_{j+1}<s\left(Q_{k}\right) \leq 1 / s_{j}\right\}
$$


where $s\left(Q_{k}\right)$ denotes the side of the supporting cube $Q_{k}$ of the atom $a_{k}$. With

$$
f_{j}(x)=\sum_{k \in I_{j}} \lambda_{k} a_{k},
$$

we can write $f$ as a sum $f=\sum_{j} f_{j}$, and we have for the sum of characteristic functions $f_{j}^{* *}=\sum_{k \in I_{j}} \lambda_{k} \chi_{Q_{k}}$ the identity $f^{* *}=\sum_{j} f_{j}^{* *}$.

We also split the space $R^{n}$ into parts $\Omega_{i}=\left\{y: s_{i-1} \leq|y|<s_{i}\right\}$. From the definition of $\left\{s_{j}\right\}$ and the condition on the weights $u$ and $v$ we get

$$
\|u\|_{L^{\frac{q_{1}}{q_{1}-q}\left(\Omega_{i}\right)}} \leq C \beta^{-q i / p} .
$$

We want to show that

$$
\left\|\hat{f}_{j}\right\|_{L_{u}^{q}\left(\Omega_{i}\right)} \leq C \beta^{-\delta|i-j|}\left\|f_{j}^{* *}\right\|_{L_{v}^{p}}
$$

with $\delta=\min \left\{1 / p,(m+1) /\left(n\left[d-\min \left(1, p / p_{1}\right)\right]\right)-1 / p\right\}$.

By Hölder's inequality we have

$$
\left\|\hat{f}_{j}\right\|_{L_{u}^{q}\left(\Omega_{i}\right)} \leq\left\|\hat{f}_{j}\right\|_{L^{q_{1}\left(\Omega_{i}\right)}}\left(\|u\|_{L^{\frac{q_{1}}{q_{1}-q}\left(\Omega_{i}\right)}}\right)^{1 / q} \leq C \beta^{-i / p}\left\|\hat{f}_{j}\right\|_{L^{q_{1}}\left(\Omega_{i}\right)} .
$$

For $j \leq i$ we use (2) and the pointwise inequality $\left|f_{j}(x)\right| \leq f_{j}^{* *}(x)$ to obtain

$$
\left\|\hat{f}_{j}\right\|_{L_{q_{1}}} \leq C\left\|f_{j}^{* *}\right\|_{L_{p_{1}}} .
$$

For $j>i$ we will make use of the fact that $f_{j}$ is a sum of atoms satisfying moment conditions. When $n=1$ this can be done by finding the antiderivative of order $m+1$ of $f_{j}$. In general, when $n \geq 1$ we proceed as follows. For each atom $a=a_{k}$ we use the following lemma.

Lemma 2. Let a be an atom with vanishing moments of order less than or equal to $m$ and with support in a cube $Q$ with side $s$. Then we have

$$
a(x)=\sum_{|\alpha|=m+1} c_{\alpha} \frac{\partial^{\alpha}}{\partial x^{\alpha}} A_{\alpha}(x),
$$

where $s^{-m-1} A_{\alpha},|\alpha|=m+1$, are atoms supported in $Q$, without vanishing moments in general, and $c_{\alpha}=2^{m+1}\left(\begin{array}{l}n \\ \alpha\end{array}\right)$.

We apply Lemma 2 to the atoms $a_{k}, k \in I_{j}$, and get the atoms $s_{j}^{m+1} A_{k, \alpha}$, $|\alpha|=m+1$. These atoms do not have vanishing moments in general. Observe that when $j>i$ we may assume that $0<s_{i}<s_{j}<\infty$ since otherwise $I_{j}$ is empty and consequently $f_{j} \equiv 0$, or $\Omega_{i}$ is the empty set. If we write $F_{j, \alpha}=$ $\sum_{k \in I_{j}}\left(s_{j}^{-m-1} \lambda_{k}\right) s_{j}^{m+1} A_{k, \alpha}$ then

$$
f_{j}(x)=\sum_{|\alpha|=m+1} c_{\alpha} \frac{\partial^{\alpha}}{\partial x^{\alpha}} F_{j, \alpha}(x),
$$


and with $f_{j}^{* *}=s_{j}^{m+1} F_{j, \alpha}^{* *}=\sum_{k \in I_{j}} \lambda_{k} \chi_{Q_{k}}$ we get

$$
\sum_{j, 0<s_{j}<\infty} s_{j}^{m+1} F_{j, \alpha}^{* *}=\sum_{j, 0<s_{j}<\infty} f_{j}^{* *} \leq \sum_{j, 0 \leq s_{j}<\infty} f_{j}^{* *}=f^{* *} .
$$

Lemma 2 is obtained by repeated use of the following lemma.

Lemma 3. Let $a$ be an atom with vanishing moments of order less than or equal to $m$ and with support in a cube $Q$ with side $s$. Then there are atoms $s^{-1} A_{l}$, $l=1, \ldots, n$, supported in $Q$ with vanishing moments of order less than or equal to $m-1$ such that

$$
a(x)=\sum_{l=1}^{n} 2 \frac{\partial}{\partial x_{l}} A_{l}(x)
$$

Proof of Lemma 3. By translation and dilation we may assume that $s=1$ and $Q=\left\{x: 0 \leq x_{i} \leq 1, i=1, \ldots, n\right\}$. Define $b_{0}(x)=a(x)$ and for $l=1, \ldots, n$, define

$$
b_{l}\left(x_{1}, \ldots, x_{n}\right)= \begin{cases}\int_{R^{l}} a\left(y_{1}, \ldots, y_{l}, x_{l+1}, \ldots, x_{n}\right) d y_{1} \cdots d y_{l} & \text { for } x \in Q \\ 0 & \text { for } x \notin Q .\end{cases}
$$

Observe that $b_{n}$ is identically zero by the moment condition of $a$. Set $a_{l}=$ $\frac{1}{2}\left(b_{l-1}-b_{l}\right)$. Then, of course, $a=2 \sum_{l=1}^{n} a_{l}$ and we claim that each function $a_{l}$ is also an atom supported in $Q$ whose moments of order less than or equal to $m$ all vanish, and furthermore that

$$
\int a_{l}\left(x_{1}, \ldots, x_{l-1}, y_{l}, x_{l+1}, \ldots, x_{n}\right) d y_{l}=0
$$

for each $\left(x_{1}, \ldots, x_{l-1}, x_{l+1}, \ldots, x_{n}\right)$. It is obvious that $a_{l}$ is supported in $Q$ and that $\left\|a_{l}\right\|_{\infty} \leq 1$. We also get (6) directly by performing the multiple integration. The moment conditions can be shown by induction as follows. Since $b_{0}=a$ we have $b_{0}$ satisfying all moment conditions of order less than or equal to $m$ by assumption. Let us assume that we have shown all moment conditions of order less than or equal to $m$ for $b_{0}, \ldots, b_{l-1}$. We will then show that $b_{l}$ satisfies all these moment conditions. Fix a monomial $x^{\alpha}$ with $|\alpha| \leq m$, and split it into parts $x^{\alpha}=h_{1}(x)+h_{2}(x)$ where $h_{1}$ is a monomial independent of $x_{l}: h_{1}(x)=\int_{0}^{1} x^{\alpha} d x_{l}=\frac{1}{\alpha_{l}+1} x_{1}^{\alpha_{1}} \cdots x_{l-1}^{\alpha_{l-1}} x_{l+1}^{\alpha_{l+1}} \cdots x_{n}^{\alpha_{n}}$, and $h_{2}(x)$ is a polynomial of degree less than or equal to $m$ satisfying $\int_{0}^{1} h_{2}(x) d x_{l}=0$. Since $b_{l}(x)$ as a function of $x_{l}$ is a multiple of the characteristic function $\chi_{[0,1]}$ for each fixed $\left(x_{1}, \ldots, x_{l-1}, x_{l+1}, \ldots, x_{n}\right)$ we get $\int_{0}^{1} b_{l}(x) h_{2}(x) d x_{l}=0$. By (6) we have $\int_{0}^{1}\left(b_{l}(x)-b_{l-1}(x)\right) h_{1}(x) d x_{l}=0$, and since by our assumption $\int b_{l-1}(x) h_{1}(x) d x=0$, we get $\int b_{l}(x) h_{i}(x) d x=0$ for $i=1,2$. Thus $b_{l}$ and hence also $a_{l}$ satisfies the moment conditions.

Finally we set

$$
A_{l}(x)=\int_{-\infty}^{x_{l}} a_{l}\left(x_{1}, \ldots, x_{l-1}, y_{l}, x_{l+1}, \ldots, x_{n}\right) d y_{l} .
$$


It is readily verified that $A_{l}$ is supported in $Q$ with $\left\|A_{l}\right\|_{\infty} \leq 1$ and, by integrating by parts, that $A_{l}$ satisfies all moment conditions of order less than or equal to $m-1$, and that $a(x)=\sum_{1}^{n} 2 a_{l}(x)=\sum_{1}^{n} 2 \partial A_{l}(x) / \partial x_{l}$.

In order to estimate the $L_{q_{1}}\left(\Omega_{i}\right)$-norm of $\hat{f}_{j}$ for $j>i$, we use HausdorffYoung's inequality (2) on the functions $F_{j, \alpha}$ together with the pointwise estimate $\left|F_{j, \alpha}(x)\right| \leq F_{j, \alpha}^{* *}(x)=s_{j}^{-m-1} f_{j}^{* *}(x)$ and get

$$
\left\|\hat{f}_{j}\right\|_{L_{q_{1}}\left(\Omega_{i}\right)} \leq C s_{i}^{m+1} \sum_{|\alpha|=m+1}\left\|\hat{F}_{j, \alpha}\right\|_{L_{q_{1}}} \leq C\left(s_{i} / s_{j}\right)^{m+1}\left\|f_{j}^{* *}\right\|_{L_{p_{1}}} .
$$

Recall that we are assuming $0<s_{i}<s_{j}<\infty$ in this case. By Lemma 1 we have $s_{i} / s_{j} \leq C \beta^{(i-j) /\left(n\left[d-\min \left(1, p / p_{1}\right)\right]\right)}$ for $j>i$. Combining the inequalities (5) and (7) we get

$$
\left\|\hat{f}_{j}\right\|_{L_{q_{1}}\left(\Omega_{i}\right)} \leq C \min \left(1, \beta^{(m+1)(i-j) /\left(n\left[d-\min \left(1, p / p_{1}\right)\right]\right)}\right)\left\|f_{j}^{* *}\right\|_{L_{p_{1}}} .
$$

In order to estimate the right-hand side of (8) by the $L_{v}^{p}$-norm of $f_{j}^{* *}$ we will use a lemma which concerns weighted norms of sums $\sum_{k} \lambda_{k} \chi_{Q_{k}}$ of characteristic functions of cubes.

Lemma 4. Let $\varphi$ be a nonnegative compactly supported function in the Schwartz class with $\varphi_{t}(x)=\varphi(x / t) t^{-n}$ and let $v$ be a weight on $R^{n}$ satisfying the doubling condition. Then for $0<p \leq p_{1}<\infty$ and for each constant $c>1$ there is a constant $C$ depending only on $c, p$ and $p_{1}$, and a constant $C_{1}$ depending only on $\varphi, p$ and $c$ such that for all $t>0$

and

$$
\left(t^{-n} \int_{R^{n}}|f(x)|^{p_{1}} d x\right)^{1 / p_{1}} \leq C\left(t^{-n} \int_{R^{n}}|f(x)|^{p} d x\right)^{1 / p}
$$

for all $f=\sum_{k} \lambda_{k} \chi_{Q_{k}}$ with $\lambda_{k}>0$ and side of $Q_{k}>t / c$ for all $k$.

The assumption that $\varphi$ has compact support is not really necessary but is just for simplicity of the proof.

Proof of Lemma 4. The lemma is a direct consequence of the following inequality [Str-T, Chapter 8, Lemma 4]:

$$
\int_{R^{n}}\left|\sum_{k} \lambda_{k} \chi_{c_{1} Q_{k}}(x)\right|^{q} v(x) d x \leq C \int_{R^{n}}\left|\sum_{k} \lambda_{k} \chi_{Q_{k}}(x)\right|^{q} v(x) d x
$$

for any constant $c_{1}>1$ with $C$ depending only on $c_{1}, p$ and $v$. To see why, we first split $R^{n}$ into a union of cubes $\tilde{Q}_{l}=\left\{x \in R^{n}: t l_{i} \leq x_{i}<t\left(l_{i}+1\right), i=\right.$ $1, \ldots, n\}, l \in Z^{n}$.

Since all cubes $Q_{k}$ have sides larger than $t / c$, then

$$
|f(x)|=\sum_{k} \lambda_{k} \chi_{Q_{k}}(x) \leq \sum_{k} \lambda_{k} \chi_{c_{1} Q_{k}}(t l) \quad \text { when } x \in \tilde{Q}_{l}
$$


and also

$$
\sum_{k} \lambda_{k} \chi_{c_{1} Q_{k}}(t l) \leq \sum_{k} \lambda_{k} \chi_{c_{2} Q_{k}}(x) \quad \text { when } x \in \tilde{Q}_{l} .
$$

(These inequalities hold if we choose $c_{1} \geq 1+2 c$ and $c_{2} \geq c_{1}+2 c$.)

From these two inequalities we get

$$
\begin{aligned}
\left(t^{-n} \int_{R^{n}}|f(x)|^{p_{1}} d x\right)^{1 / p_{1}} & \leq\left(\sum_{l \in Z^{n}}\left(\sum_{k} \lambda_{k} \chi_{c_{1} Q_{k}}(t l)\right)^{p_{1}}\right)^{1 / p_{1}} \\
& \leq\left(\sum_{l \in Z^{n}}\left(\sum_{k} \lambda_{k} \chi_{c_{1} Q_{k}}(t l)\right)^{p}\right)^{1 / p} \\
& \leq\left(t^{-n} \int_{R^{n}}\left(\sum_{k} \lambda_{k} \chi_{c_{2} Q_{k}}(x)\right)^{p} d x\right)^{1 / p} \\
& \leq C\left(t^{-n} \int_{R^{n}}|f(x)|^{p} d x\right)^{1 / p}
\end{aligned}
$$

where we have used the result of [Str-T] to obtain the last inequality. Since

$$
\int_{R^{n}}|f(x)|^{p} \varphi_{t} * v(x) d x=\int_{R^{n}} \varphi_{t}(y)\left(\int_{R^{n}}|f(x+y)|^{p} v(x) d x\right) d y
$$

and

$$
|f(x+y)| \leq \sum_{k} \lambda_{k} \chi_{c_{3} Q_{k}}(x)
$$

if $\varphi_{t}(y) \neq 0$ and we choose $c_{3}$ such that $c_{3} \geq 1+2 c_{4} c$ where $\operatorname{supp} \varphi \subset\{|y| \leq$ $\left.c_{4}\right\}$, we also get

$$
\begin{aligned}
\int_{R^{n}}|f(x)|^{p} \varphi_{t} * v(x) d x & \leq\left(\int \varphi_{t} d y\right)\left(\int_{R^{n}}\left(\sum_{k} \lambda_{k} \chi_{c_{3} Q_{k}}(x)\right)^{p} v(x) d x\right) \\
& \leq C_{1} \int_{R^{n}}|f(x)|^{p} v(x) d x .
\end{aligned}
$$

This completes the proof of Lemma 4.

Remark. We do not need the last part of the lemma, which uses the doubling condition of $v$, if we replace the inequalities in Proposition 1 by

$$
\|\hat{f}\|_{L_{u}^{q}} \leq C\left\|f^{* * *}\right\|_{L_{v}^{p}} \text { and }\|\hat{f}\|_{L_{u}^{q}} \leq C\left\|f^{* r * *}\right\|_{L_{v}^{p}} \quad \text { resp. }
$$

where $f^{* * *}=\sum_{k} \lambda_{k} \chi_{c_{3} Q_{k}}$ and $f^{* r * *}=\left(\sum_{k} \lambda_{k}^{r} \chi_{c_{3} Q_{k}}\right)^{1 / r}$ As we shall see in $\S 6$, these somewhat weaker inequalities will be sufficient to prove Theorem 1.

The function $f_{j}^{* *}$ is the finite sum of characteristic functions of cubes $Q_{k}$, $k \in I_{j}$. We may assume that $I_{j}$ is not the empty set. Let

$$
\bar{s}_{j}=1 / \min _{k \in I_{j}} s\left(Q_{k}\right)
$$


Thus $s_{j} \leq \bar{s}_{j} \leq s_{j+1}$ and $0<\bar{s}_{j}<\infty$. The function $f_{j}^{* *}$ is a sum of characteristic functions of cubes with sides larger than $1 / \bar{s}_{j}$. In the case $p \leq p_{1}$ we get by the definition of $s_{j}$ the inequality

$$
\bar{s}_{j}^{n\left(1 / p-1 / p_{1}\right)} \beta^{-j / p} \leq C\left(\inf _{x \in R^{n}} \varphi_{1 / \bar{s}_{j}} * v(x)\right)^{1 / p} .
$$

By this inequality and the first inequality in Lemma 4 we get in the case $p \leq p_{1}$

$$
\left\|f_{j}^{* *}\right\|_{p_{1}} \leq C\left(\bar{s}_{j}\right)^{n\left(\frac{1}{p}-\frac{1}{p_{1}}\right)}\left\|f_{j}^{* *}\right\|_{p} \leq C \beta^{\frac{1}{p}}\left(\int_{R^{n}}\left|f_{j}^{* *}(x)\right|^{p} \varphi_{1 / s_{j}} * v(x) d x\right)^{\frac{1}{p}} .
$$

Similary, in the case when $p>p_{1}$ we get by Hölder's inequality

$$
\begin{aligned}
\left\|f_{j}^{* *}\right\|_{p_{1}} & \leq\left(\int_{R^{n}}\left(\varphi_{1 / \bar{s}_{j}} * v\right)^{-\frac{p_{1}}{p-p_{1}}} d x\right)^{\frac{p-p_{1}}{p_{1} p}}\left(\int_{R^{n}}\left|f_{j}^{* *}\right|^{p} \varphi_{1 / \bar{s}_{j}} * v d x\right)^{\frac{1}{p}} \\
& \leq C \beta^{j / p}\left(\int_{R^{n}}\left|f_{j}^{* *}(x)\right|^{p} \varphi_{1 / \bar{s}_{j}} * v(x) d x\right)^{\frac{1}{p}} .
\end{aligned}
$$

By the second inequality in Lemma 4, we conclude in both cases that

$$
\left\|f_{j}^{* *}\right\|_{p_{1}} \leq C \beta^{j / p}\left\|f_{j}^{* *}\right\|_{L_{v}^{p}}
$$

By (4), (8), and (9) we have

$$
\left\|\hat{f}_{j}\right\|_{L_{u}^{q}\left(\Omega_{i}\right)} \leq C \beta^{(j-i) / p} \min \left(1, \beta^{(m+1)(i-j) /\left(n\left[d-\min \left(1, p / p_{1}\right)\right]\right)}\right)\left\|f_{j}^{* *}\right\|_{L_{v}^{p} .}
$$

From this we get (3) for any

$$
0<\delta \leq \min \left\{1 / p,(m+1) /\left(n\left[d-\min \left(1, p / p_{1}\right)\right]\right)-1 / p\right\}
$$

provided $m+1>n\left[d-\min \left(1, p / p_{1}\right)\right] / p$.

\section{Summation of all Pieces}

In order to prove Proposition 1 it remains only to put the pieces together again using estimate (3), which was

$$
\left\|\hat{f}_{j}\right\|_{L_{u}^{q}\left(\Omega_{i}\right)} \leq \beta^{-\delta|i-j|}\left\|f_{j}^{* *}\right\|_{L_{v}^{p}}
$$

for some $\delta>0$. Let $q_{0}=\min (q, 1)$. By Minkowski's inequality or the triangle inequality we have

$$
\|\hat{f}\|_{L_{u}^{q}\left(\Omega_{i}\right)}^{q_{0}} \leq \sum_{j}\left\|\hat{f}_{j}\right\|_{L_{u}^{q}\left(\Omega_{i}\right)}^{q_{0}},
$$

and since the sets $\Omega_{i}, i=\ldots,-1,0,1, \ldots$, are disjoint we also have

$$
\|\hat{f}\|_{L_{u}^{q}} \leq\left(\sum_{i}\|\hat{f}\|_{L_{u}^{q}\left(\Omega_{i}\right)}^{q}\right)^{1 / q} .
$$


Thus by combining inequalities, we get

$$
\|\hat{f}\|_{L_{u}^{q}} \leq C\left(\sum_{i}\left(\sum_{j} \beta^{-q_{0} \delta|i-j|}\left\|f_{j}^{* *}\right\|_{L_{v}^{p}}^{q_{0}}\right)^{q / q_{0}}\right)^{1 / q} .
$$

Let $0<r \leq 1$. Then

$$
\sum_{j}\left(f_{j}^{* *}(x)\right)^{r}=\sum_{j}\left(\sum_{k \in I_{j}} \lambda_{k} \chi_{Q_{k}}(x)\right)^{r} \leq \sum_{k} \lambda_{k}^{r} \chi_{Q_{k}}(x)=\left(f^{* r *}(x)\right)^{r} .
$$

Let $p_{0}=\max (p, r)$. We use the pointwise inequality

$$
\sum_{j}\left(f_{j}^{* *}(x)\right)^{p} \leq\left(\sum_{j}\left(f_{j}^{* *}(x)\right)^{r}\right)^{p / r}
$$

when $p \geq r$ and the concavity of the $L_{v}^{p / r}$-'norm' for positive functions (i.e., the inequality opposite to the usual Minkowski inequality) when $0<p \leq r$ to obtain the inequality

$$
\left(\sum_{j}\left(\left\|f_{j}^{* *}\right\|_{L_{r}^{p}}\right)^{p_{0}}\right)^{1 / p_{0}} \leq\left\|\left(\sum_{j}\left(f_{j}^{* *}\right)^{r}\right)^{1 / r}\right\|_{L_{v}^{p}} \leq\left\|f^{* r *}\right\|_{L_{v}^{p}} .
$$

To complete the proof of Proposition 1 we need only to show that the right side of (10) is less than the left side of (11). To do this, we will apply the following discrete version of Hardy's inequality with the exponents $r_{0}=q, r_{1}=q_{0}$ and $r_{2}=p_{0}$.

Lemma 5. Let $0<r_{1}<\infty, 0<r_{2}<\infty, r_{0} \geq r_{2}, \beta>1$, and $\delta>0$. Then

$$
\left(\sum_{i}\left(\sum_{j} \beta^{-\delta|i-j|} a_{j}^{r_{1}}\right)^{r_{0} / r_{1}}\right)^{1 / r_{0}} \leq C\left(\sum_{j} a_{j}^{r_{2}}\right)^{1 / r_{2}}
$$

for any sequence $\left\{a_{j}\right\}_{-\infty}^{\infty}$ of nonnegative numbers.

Proof of Lemma 5. Using Hölder's inequality on the inner sum in the case $r_{1}<r_{2}$ we get

$$
\begin{aligned}
\sum_{j} \beta^{-\delta|i-j|} a_{j}^{r_{1}} & \leq\left(\sum_{j} \beta^{-\delta|i-j|} a_{j}^{r_{2}}\right)^{r_{1} / r_{2}}\left(\sum_{j} \beta^{-\delta|i-j|}\right)^{1-r_{1} / r_{2}} \\
& \leq C\left(\sum_{j} \beta^{-\delta|i-j|} a_{j}^{r_{2}}\right)^{r_{1} / r_{2}} .
\end{aligned}
$$


For $r_{1} \geq r_{2}$, Hölder's inequality is replaced by the inequality

$$
\sum_{j} b_{j}^{r_{1}} \leq\left(\sum_{j} b_{j}^{r_{2}}\right)^{r_{1} / r_{2}}
$$

for $b_{j} \geq 0$. Using the inequality

$$
\left(\sum_{i} b_{i}^{r_{0} / r_{2}}\right)^{1 / r_{0}} \leq\left(\sum_{i} b_{i}\right)^{1 / r_{2}}
$$

for $b_{i} \geq 0$, we see that there is a $\delta_{1}>0$ such that the left-hand side of the inequality in Lemma 5 is bounded by

$$
\leq C\left(\sum_{i} \sum_{j} \beta^{-\delta_{1}|i-j|} a_{j}^{r_{2}}\right)^{1 / r_{2}} \leq C\left(\sum_{j} a_{j}^{r_{2}}\right)^{1 / r_{2}} .
$$

This completes the proof of Lemma 5.

Combining Lemma 5 with (10) and (11) we get

$$
\|\hat{f}\|_{L_{u}^{q}} \leq C\left\|f^{* r *}\right\|_{L_{v}^{p}}
$$

This completes the proof of Proposition 1.

\section{LIMITING ARGUMENTS}

In this section we are going to use the estimate for the Fourier transform of a finite sum of atoms to obtain a similar estimate for functions in $\mathscr{S}_{0,0}$, and then we will extend that estimate to all tempered distributions in $H_{v}^{p}$.

If $f$ is a function in $\mathscr{S}_{0,0}$ then it can be written as an infinite sum of atoms, i.e., $f=\sum_{k=1}^{\infty} \lambda_{k} a_{k}$ (with $\lambda_{k}>0$ ) such that the partial sums $f_{N}=\sum_{k=1}^{N} \lambda_{k} a_{k}$ converge pointwise to $f$ almost everywhere. Furthermore, for any $m \geq 0$, $M>0$ and $r>0$, the decomposition can be done such that

$$
f^{* r *}(x)=\left(\sum_{k} \lambda_{k}^{r} \chi_{B_{k}}(x)\right)^{1 / r} \leq C_{M, m, r} f^{* M}(x) \text { for all } x,
$$

where $B_{k}$ is a supporting ball for the atom $a_{k}$ and with the atoms $a_{k}$ satisfying all moment conditions of order less than or equal to $m$ (see [Str-T]). (In fact, by careful use of the Whitney decomposition of the sets $\left\{f^{* M}>2^{l}\right\}$ when doing the atomic decomposition we may replace $\chi_{B_{k}}$ by $\chi_{c B_{k}}$ for any fixed $c>0$ in the inequality above.) For any $\lambda>0$ and any $\psi$ in $\mathscr{S}$ with nonzero integral, the right-hand side is dominated by $C N_{\psi}^{\lambda} f(x)$ provided $M$ is large enough depending on $\lambda$.

For all $\lambda>0$ and all $f \in \mathscr{S}_{0,0}$ we have

$$
N_{\psi}^{\lambda} f(x) \leq C_{\lambda, f, \psi}(1+|x|)^{-\lambda}, \quad x \in R^{n}
$$


If $\lambda>n$ the finite sums of atoms $f_{N}$ are dominated by $C_{\lambda, f, \psi}(1+|x|)^{-\lambda} \in L^{1}$, and hence the $f_{N}$ converge to $f$ in $L^{1}$ norm by the dominated convergence theorem. Thus the Fourier transforms $\hat{f}_{N}(y)$ converge to $\hat{f}(y)$ uniformly for all $y \in R^{n}$. Since

$$
\left\|\hat{f}_{N}\right\|_{L_{u}^{q}} \leq C\left\|f_{N}^{* r *}\right\|_{L_{v}^{p}} \leq C\left\|N_{\psi}^{\lambda} f\right\|_{L_{v}^{p}}
$$

by Proposition 1, provided $0<r \leq \min (q, 1)$, we conclude that

$$
\|\hat{f}\|_{L_{u}^{q}} \leq C\left\|N_{\psi}^{\lambda} f\right\|_{L_{v}^{p}} \text { for } f \in \mathscr{S}_{0,0} .
$$

Next assume that $f$ is a tempered distribution in $H_{v}^{p}$. Since $\mathscr{S}_{0,0}$ is dense in $H_{v}^{p}$, we get a sequence of functions $f_{N} \in \mathscr{S}_{0,0}$ which converges to $f$ in $H_{v}^{p}$ norm and hence also in the distributional sense. We may assume that $\left\|f_{N}-f_{N-1}\right\|_{H_{v}^{p}} \leq 2^{-N}$. We may assume that $\int\left|\varphi_{1 / s} * v\right|^{-p_{1} /\left(p-p_{1}\right)} d x<\infty$ if $p>p_{1}$ (resp., $\inf _{x \in R^{n}}\left|\varphi_{1 / s} * v\right| \geq c>0$ when $p \leq p_{1}$ ), since otherwise the $P W\left(q, p ; q_{1}, p_{1}\right)$ condition would imply that $u$ is identically zero. If $u_{1}(x)=$ $(1+|x|)^{-l}$, then it follows from Lemma 1 that the pair of weights $\left(u_{1}, v\right)$ satisfies the $P W\left(q, p ; q_{1}, p_{1}\right)$ condition provided $l$ is chosen large enough. By the estimate above of the Fourier transform of a function in $\mathscr{S}_{0,0}$ it follows that $\left\{\hat{f}_{N}\right\}$ is a Cauchy sequence in $L_{u_{1}}^{q}$ and hence converges in norm to a function $g$ in $L_{u_{1}}^{q}$. Since the weight $u_{1} \geq c>0$ on compact sets, $\hat{f}_{N}$ converges to $\mathrm{g}$ in measure on compact sets. The sequence $\left\{\hat{f}_{N}\right\}$ is also a Cauchy sequence in $L_{u}^{q}$ and since $\hat{f}_{N}$ converges in measure to $g$ on compact sets we conclude that $\hat{f}_{N}^{u}$ converges to $g$ in $L_{u}^{q}$ norm and that

$$
\|g\|_{L_{u}^{q}}=\lim _{N \rightarrow \infty}\left\|\hat{f}_{N}\right\|_{L_{u}^{q}} \leq C \lim _{N \rightarrow \infty}\left\|f_{N}\right\|_{H_{v}^{p}}=C\|f\|_{H_{v}^{p}} .
$$

It remains to show that the tempered distribution $\hat{f}$ is obtained from the function $g$, i.e., to show that

$$
\langle\hat{f}, \phi\rangle=\int_{R^{n}} g(x) \phi(x) d x \quad \text { for all } \phi \in \mathscr{S} .
$$

Since $f_{N}$ converges to $f$ in the distributional sense we have

$$
\langle\hat{f}, \phi\rangle=\langle f, \hat{\phi}\rangle=\lim _{N \rightarrow \infty}\left\langle f_{N}, \hat{\phi}\right\rangle=\lim _{N \rightarrow \infty}\left\langle\hat{f}_{N}, \phi\right\rangle,
$$

and since $\phi \in \mathscr{S}$, which is contained in the dual space of $L_{u_{1}}^{q}$, and also $\hat{f}_{N}$ converges to $g$ in $L_{u_{1}}^{q}$, we conclude that

$$
\lim _{N \rightarrow \infty}\left\langle\hat{f}_{N}, \phi\right\rangle=\int_{R^{n}} g(x) \phi(x) d x .
$$

Thus we have shown that

$$
\langle\hat{f}, \phi\rangle=\int_{R^{N}} g(x) \phi(x) d x
$$


with

$$
\|g\|_{L_{u}^{q}} \leq C\|f\|_{H_{v}^{p}}
$$

This completes the proof of Theorem 1 .

\section{REFERENCES}

[A] E. Adams, On the identification of weighted Hardy spaces, Indiana Univ. Math. J. 32 (1983), 477-489.

[B-H] J. J. Benedetto and H. Heinig, Fourier transform inequalities with measure weights, Adv. in Math. (to appear).

[B-H-J] J. J. Benedetto, H. Heinig and R. Johnson, Fourier inequalities with $A_{p}$ weights, General Inequalities, 5 (W. Walter, ed.), Internat. Ser. Numer. Math. 80 (1987), 217-232.

[H1] H. Heinig, Weighted norm inequalities for classes of operators, Indiana Univ. Math. J. 33 (1984), 573-582.

[H2] H. Heinig, Fourier operators on weighted Hardy spaces, Proc. Cambridge Philos. Soc. 101 (1987), 113-121.

[J-Sam] W. B. Jurkat and G. Sampson, On rearrangement and weight inequalities for the Fourier transform, Indiana Univ. Math. J. 33 (1984), 257-267.

[M] B. Muckenhoupt, Weighted norm inequalities for the Fourier transform, Trans. Amer. Math. Soc. 176 (1983), 729-742.

[Sad-W] C. Sadosky and R. L. Wheeden, Some weighted norm inequalities for the Fourier transform of functions with vanishing moments, Trans. Amer. Math. Soc. 300 (1987), 521-533.

[St] E. M. Stein, Interpolation of linear operators, Trans. Amer. Math. Soc. 83 (1956), 482-492.

[Str-T] J.-O. Strömberg and A. Torchinsky, Weighted Hardy Spaces, Lecture Notes in Math., vol. 1381, Springer, 1989.

[Str-W] J.-O. Strömberg and R. L. Wheeden, Relations between $H_{u}^{p}$ and $L_{u}^{p}$ with polynomial weights, Trans. Amer. Math. Soc. 270 (1982), 439-467.

Institute of Mathematical and Physical Sciences, University of Tromsø, N-9001 TROMS $\emptyset$, NORWAY

Department of Mathematics, Rutgers University, New Brunswick, New Jersey 08903 